PREHOSPITAL CARE

\title{
The optimum reperfusion pathway for ST elevation acute myocardial infarction: development of a decision framework
}

\section{J Kendall}

Emerg Med J 2007;24:52-56. doi: 10.1136/emj.2006.042952

There is currently much debate about the relative roles of pharmacological reperfusion (ie, thrombolysis) and mechanical reperfusion (ie, primary percutaneous coronary intervention (PPCl) in the management of patients with acute ST segment elevation acute myocardial infarction (STEMI). Whilst the scientific debate is reaching some resolution in terms of appropriate interpretation of the evidence base, there are still significant resource issues within the UK that limit our ability to implement gold standard reperfusion therapy.

Current evidence supports the use of one or other strategy in certain situations depending on various patient-related and logistical factors. This paper reviews the literature and builds the case for developing a strategic approach which includes both mechanical and pharmacological interventions, proposing that these are not mutually exclusive-indeed, that an approach which excludes one of these interventions will not be to the benefit of all patients. There is also a discussion of the role of rescue $\mathrm{PPCl}$, facilitated $\mathrm{PPCl}$ and early post thrombolysis angiography in the management of STEMI.

Cardiac networks throughout the UK are developing strategies to improve access to these interventions and this paper offers advice on the logical selection of interventions for reperfusion in the context of a clinical decision framework that is evidencebased, pragmatic and develops through a series of scenarios with increasing availability of resources. Four sequential scenarios are presented: the first to set the scene is largely consigned to history; the last, as of yet, is not robustly achievable within the UK, but represents the "optimum reperfusion pathway", to which most cardiac networks are striving. Most of us currently find ourselves in a period of change between the two and will relate to either scenario two or three.

\section{Correspondence to: Dr J Kendall, Emergency Department, Frenchay Hospital, North Bristol NHS Trust, Frenchay Park Road, Bristol BS16 1LE, UK; jason.kendall@nbt.nhs.uk}

Accepted 12 October 2006
U ntil relatively recently, the debate over the best approach to adopt for the emergency treatment of a patient with ST elevation myocardial infarction (STEMI) was largely theoretical, occupying much space in specialist journals but, because of resource limitations, of little practical relevance to those at the front line of emergency medicine and cardiac care. Although there is impressive evidence to show the superiority of primary percutaneous coronary intervention (PPCI) over in-hospital thrombolysis, the paucity of PPCI provision across large expanses of the UK has meant that few healthcare systems were in a position to put this knowledge into practice. In addition, the lack of robust head-tohead comparisons between PPCI and the rapidly emerging technique of prehospital thrombolysis (PHT) has made it difficult to decide in which direction the emergency STEMI service should progress. The use of rapid "point of presentation" emergency department based thrombolysis protocols has also confounded the issue, as it seems unlikely that previous trials with thrombolysis delivered by infusions (often associated with major delays) bear much relation to the more modern, rapid administration of bolus thrombolytics.

Emergency care of patients with STEMI is now changing rapidly. An expansion in both the provision of PPCI and in the implementation of PHT has resulted in cardiac networks around the country choosing to incorporate either or both techniques into their protocols for the emergency care of STEMI. In-hospital thrombolysis also continues to improve as the use of robust emergency department-based protocols and prehospital alerting or electrocardiograph telemetry produces ever-decreasing door-to-needle times. These in-hospital processes of care will always continue to be required for the proportion of patients who self-present to the emergency department with chest pain.

As of July 2006, of the UK's 31 ambulance services, 28 were trained to diagnose STEMI and administer thrombolysis to appropriate patients before their arrival at hospital. ${ }^{1}$ The technique has already been used in about 5000 patients, almost half of whom received it in 2005-6. The Myocardial Infarction National Audit Project reported that in 2005-6, 1647 patients were treated with PPCI in preference to thrombolytic treatment, compared with 1087 in 2004-5..$^{2}$ In-hospital thrombolysis in $88 \%$ of hospitals in the UK is now delivered to $75 \%$ of eligible patients within 30 min of their arrival at hospital. ${ }^{2}$

PHT, rapid in-hospital thrombolysis and PPCI are all potentially life-saving techniques, and their inclusion into emergency care protocols is to be welcomed. However, with different healthcare

Abbreviations: PHT, prehospital thrombolysis; PPCl, primary percutaneous coronary intervention; STEMI, ST elevation myocardial infarction 
economies expanding their protocols in different ways and at different rates, there is a danger that disparities may occur. If we are to avoid a postcode lottery, then there is a need for guidance to ensure that healthcare economies develop their protocols in a systematic fashion that corresponds to the latest evidence base.

\section{EVIDENCE-BASED EMERGENCY STEMI CARE}

Current evidence suggests that PPCI after STEMI offers better short-term mortality, reduced reinfarction rates and a lower incidence of stroke than hospital-delivered thrombolysis. ${ }^{3}$ PPCI also provides useful anatomical information that can be used to determine the patient's subsequent management. It will identify patients with severe three-vessel disease, severe left main coronary artery stenosis or occluded vessels unsuitable for angioplasty, who can then be referred for bypass surgery. Investing in PCI facilities also allows "rescue PCI" for patients with clinical or electrocardiographic failed reperfusion after thrombolysis. ${ }^{45}$ The government is currently investigating seven different service models of PPCI in different geographical settings. ${ }^{2}$ The results are awaited with interest.

In the meantime, however, healthcare systems should be cautioned against a single-minded focus on PPCI. Although the development of a PPCI service accessible $24 \mathrm{~h}$ a day is laudable, the current evidence base would not support its use for every patient all of the time, and particularly for patients who present very early after the onset of symptoms.

The evidence base specifically comparing PPCI with PHT is limited. The only study specifically examining this issue showed no important outcome benefit associated with PPCI. ${ }^{6}$ In particular, current evidence would suggest that the benefits of PPCI over thrombolysis are at their least robust early in the course of the evolution of an infarct and especially within $2 \mathrm{~h}$ of the onset of symptoms. ${ }^{7}$ A large proportion of patients in the UK present within this time window, particularly in urban and suburban environments. ${ }^{2}$ Regarding the delay to PPCI when compared with thrombolysis ("balloon time" minus "needle time"), this benefit may become a harm when a period of $60 \mathrm{~min}$ is exceeded. ${ }^{8}$ Such ready access to PPCI facilities is not always possible.

Catheterisation laboratories are expensive to set up, incur substantial running costs and must be staffed $24 \mathrm{~h}$ a day and seven days a week for a robust PPCI service to be viable. In many areas, the patient load will be insufficient to justify such an investment, especially as it is necessary for PCI centres to operate at high volume simply to provide the staff with the necessary experience to develop and maintain their expertise. It is recognised that there are increased delays associated with PPCI performed at night compared with during the day, ${ }^{9}$ and adverse outcome in centres with a low volume of cases compared with that in centres with higher case loads. ${ }^{10}$

The above issues have led the European Society of Cardiology and the American College of Cardiology/American Heart Association to give specific guidance for the recommendation of PPCI in patients with STEMI. ${ }^{11-14}$ Specifically, thrombolysis is generally preferred if "early presentation (within three hours of onset of symptoms) and delay to invasive strategy (balloon minus needle time) is greater than 60 minutes"; an invasive strategy is generally preferred if "skilled PCI laboratory is available (balloon minus needle time is less than 60 minutes), or late presentation (symptom onset is more than three hours ago)"; there is no preference for either strategy if presentation is less than $3 \mathrm{~h}$ and there is no delay to an invasive strategy. ${ }^{14}$ Patients also benefit from PPCI if they have a high-risk STEMI (eg, presence of cardiogenic shock $)^{15}$ or a clear contraindication to thrombolysis (eg, recent haemorrhagic stroke).
Therefore, the evidence base would suggest that there is a defined place for the delivery of expedient thrombolysis, and particularly PHT, in certain groups of patients and in certain circumstances. Immediate thrombolysis is recommended in patients who present early (within $3 \mathrm{~h}$ of the onset of symptoms) and in whom delay to PPCI is likely to exceed $60 \mathrm{~min}$, and in situations where PPCI is simply not available. The prehospital delivery of a bolus thrombolytic by a paramedic properly trained in the use of 12-lead electrocardiogram equipment can save valuable time in the treatment of patients with STEMI. Evidence of the benefit of PHT over in-hospital thrombolysis is unequivocal: it has been estimated that PHT offers a survival benefit (over in-hospital thrombolysis) of $17 \%$ in patients with acute STEMI. ${ }^{16}$

Patients who do not fulfil specific criteria for $\mathrm{PHT}^{17}$ or who present directly to the emergency department (in situations where PPCI is not readily available) will still require rapid inhospital thrombolysis; encouragingly, the National Service Framework standards for door-to-needle times are certainly achievable when emergency department-based systems are robust. $^{2}$

It is a vital part of any pharmacological reperfusion strategy that potential reocclusion is actively considered and dealt with by "rescue" mechanical intervention. ${ }^{45}$ However, irrespective of the apparent success of thrombolysis, good evidence is now emerging for predischarge angiography ${ }^{18} 19$ and is a recommendation of the most recent European Society of Cardiology Taskforce Guidance. ${ }^{11}$ Thus, thrombolysis, even if successful, should not be considered the final treatment: "lyse now, stent later". ${ }^{20}$ The intuitive advantages of this strategy are that it combines the benefits of early pharmacological reperfusion with the benefits of PCI (reduced reocclusion, anatomical definition of pathology, etc) performed in a more semi-elective environment, when outcome is known to be improved. This should not be confused with the concept of "facilitated PCI", which is planned immediate PCI after an initial pharmacological regimen, ${ }^{12}{ }^{14}$ for which there is, according to our knowledge, no robust clinical outcome data to show that benefit outweighs risk. ${ }^{21}$

\section{CHOICE OF THE THROMBOLYTIC AGENT}

The National Institute for Health and Clinical Excellence recommends that, in hospital, the choice of the thrombolytic drug should take account of the following: ${ }^{22}$

- The likely balance of benefit and harm

- UK clinical practice, in which patients who have previously received streptokinase should not be treated with it again

- The hospital's arrangements for reducing delays in the administration of thrombolysis

Table 1 Possible combinations of available interventions in different resource environments

\begin{tabular}{llll}
\hline $\begin{array}{l}\text { In-hospital } \\
\text { thrombolysis }\end{array}$ & $\begin{array}{l}\text { Pre-hospital } \\
\text { thrombolysis }\end{array}$ & $\begin{array}{l}\text { Primary } \\
\text { percutaneous } \\
\text { coronary } \\
\text { intervention }\end{array}$ & $\begin{array}{l}\text { Rescue } \\
\text { percutaneous } \\
\text { coronary } \\
\text { intervention }\end{array}$ \\
\hline Yes & No & No & No \\
Yes & No & No & $+/-$ \\
Yes & $+/-$ & No & $+/-$ \\
Yes & Yes & No & $+/-$ \\
Yes & Yes & $+/-$ & $+/-$ \\
Yes & Yes & $+/-$ & Yes \\
Yes & Yes & Yes & Yes \\
\hline
\end{tabular}

Resources, interventions and therapeutic options improving from top to bottom of table. 


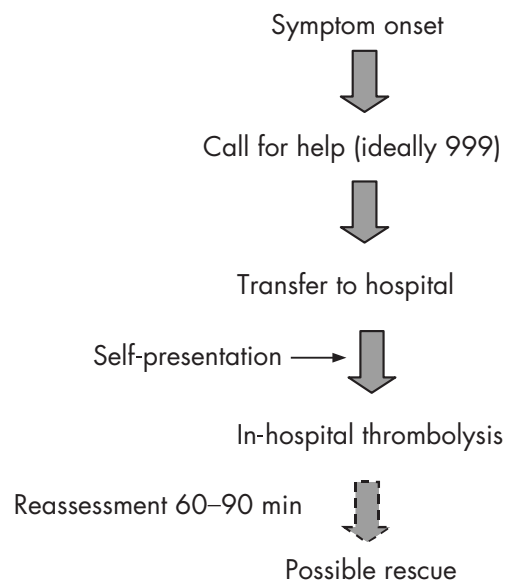

Figure 1 Stage 1 in the evolution of an emergency ST elevation myocardial infarction protocol. No pre-hospital thrombolysis (PHT), no primary percutaneous coronary intervention (PPCI), limited rescue percutaneous coronary intervention (PCI).

In prehospital care:

- The choice of thrombolytic should be restricted to the bolus agents tenecteplase or reteplase

The Coronary Heart Disease Collaborative included bolus drugs among its recommended "effective approaches that have improved practice" as part of fast-track protocols in the inhospital setting. ${ }^{23}$ Where delivery is in the prehospital setting, the practicalities of administration mean that bolus drugs are the preferred option.

\section{DEVELOPMENT OF A DECISION FRAMEWORK FOR THE MANAGEMENT OF PATIENTS WITH ST ELEVATION AMI}

This section attempts to build an "optimum reperfusion pathway" using a practical tool that combines best evidence, local resource provision and a pragmatic approach that facilitates the decision-making process to improve performance in the delivery of care to patients with STEMI.

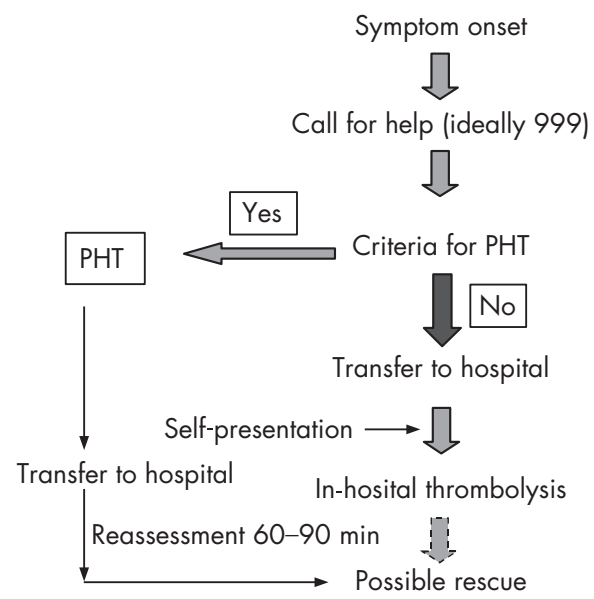

Figure 2 Stage 2 in the evolution of an emergency ST elevation myocardial infarction protocol. Pre-hospital thrombolysis (PHT), no primary percutaneous coronary intervention (PPCI), limited rescue percutaneous coronary intervention (PCI).
The aim is to help map the current situation and model of care provided in a healthcare economy, review where improvements need to be made and determine the necessary changes required to move to an improved model of care. The resulting decision framework offers a staged progression through four different scenarios based on the relative availability of resources and infrastructure.

In doing this it is necessary to make several assumptions. Crucially, and consistent with the current evidence base, the decision framework regards pharmacological and mechanical approaches to reperfusion as complimentary strategies depending on individual patient circumstances and available resource. It is also assumed that in-hospital thrombolysis delivery is now universally robust, and that PHT and access to rescue PCI are likely to be available before robust PPCI-assumptions that are largely consistent with the current and developing resource situation in the UK.

Table 1 shows several possible combinations of interventions that may be available in a particular healthcare economy. Each one represents a certain resource scenario, with the poorest available resources occurring at the top of the table and the "nirvana" of healthcare for STEMI (where all interventions are robustly available) occurring at the bottom. Cardiac networks should be aiming to move down the table as resources improve, new interventions are implemented and more therapeutic options are available. This will permit best application of the evidence base, best reperfusion strategies and best outcome for patients.

The decision framework is essentially a series of clinical decisions that builds through a series of stages as resources improve, resulting in what could be considered an optimum pathway. The development of the decision framework is exemplified by its application to scenarios with ever-increasing intervention options.

Stage 1: no $\mathrm{PHT}$, no $\mathrm{PPCl}$, limited rescue $\mathrm{PCl}$

This represents the most basic stage of the decision framework where there are essentially no intervention options other than in-hospital thrombolysis and therefore no choices available to the clinician (fig 1). This scenario was almost universal within the UK until recently. However, given the unequivocal evidence supporting PHT to in-hospital thrombolysis, this situation has been largely consigned to history.

\section{Stage 2: $\mathrm{PHT}$, no $\mathrm{PPCl}$, limited rescue $\mathrm{PCI}$}

The implementation of PHT has moved the decision framework to the next stage as a therapeutic option has been introduced (fig 2). This is currently a common scenario in the UK. Patients can be selected for PHT on the basis of predefined eligibility criteria.

However, although it satisfies the evidence base supporting PHT to in-hospital thrombolysis, there is no provision for PPCI, which is to the detriment of a large number of patients. The priority for a cardiac network in this situation is the initial development of at least a limited PPCI service, which will allow this therapeutic option to be considered in certain patients at certain times, taking the decision framework on to a further stage.

\section{Stage 3: PHT, limited $\mathrm{PPCl}$, rescue $\mathrm{PCI}$}

This is an ever-increasing scenario in the UK and begins to deliver options that most closely satisfy the current evidence base (fig 3). Where availability, time, traffic or geography stands in the way of rapid delivery of PPCI, the responsible clinician (whether a paramedic in the prehospital setting or a doctor in hospital) will have several questions to ask: (1) is PPCI available and (2) does the patient fulfil criteria for PPCI rather than PHT (ie, what is the time since the onset of 


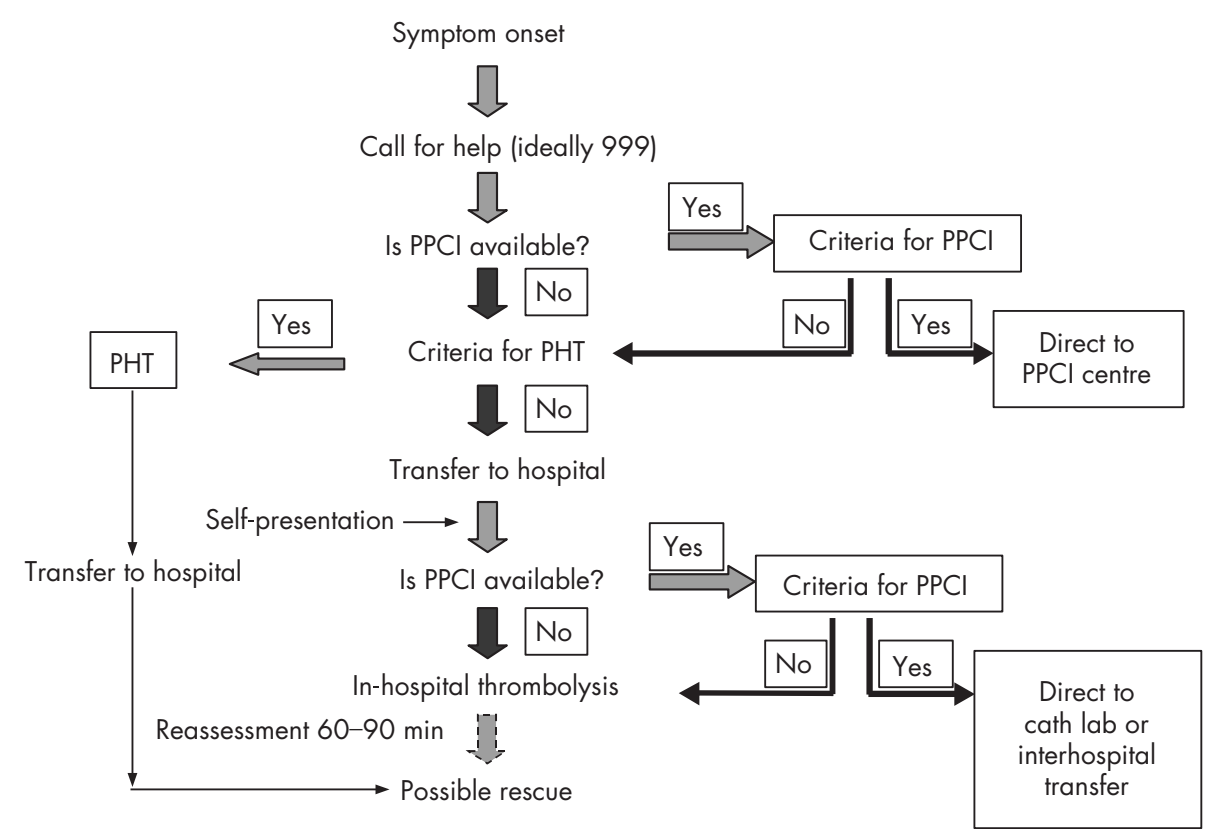

Figure 3 Stage 3 in the evolution of an emergency ST elevation myocardial infarction protocol. Pre-hospital thrombolysis (PHT), limited primary percutaneous coronary intervention (PPCI), rescue percutaneous coronary intervention (PCI).

symptoms, what is the potential delay to PPCI and is there the presence of shock or contraindications to thrombolysis?) Figure 3 shows these decision points and allow the decision framework to mature further.

Important practical issues need to be considered: will it be an extension of the role of the paramedic to determine which strategy is best for individual patients? What telemetry and communication systems can be put in place to support the decision-making process? Who takes responsibility for the decision? Is there an infrastructure in place to allow direct access of a patient from the ambulance to the catheterisation laboratory, or is access only via the emergency department or the coronary care unit? Clearly, it would seem advantageous if the patient could be delivered directly to the catheterisation laboratory from the prehospital setting. Irrespective of the potential for this access, there will always be a need for a decision point in the emergency department for patients who present directly to hospital.

\section{Stage 4: robust $\mathrm{PHT}, \mathrm{PPCl}$, rescue $\mathrm{PCI}$}

This represents the optimum reperfusion pathway, and is the final stage in the development of the decision framework, delivering therapeutic options that most closely satisfy the current evidence base and allowing decisions to be based largely on clinical and logistic, not resource, criteria (fig 4). This situation is currently rare in the UK but should be the longterm aim of cardiac networks and the broader healthcare community. At this final stage in the framework, availability of

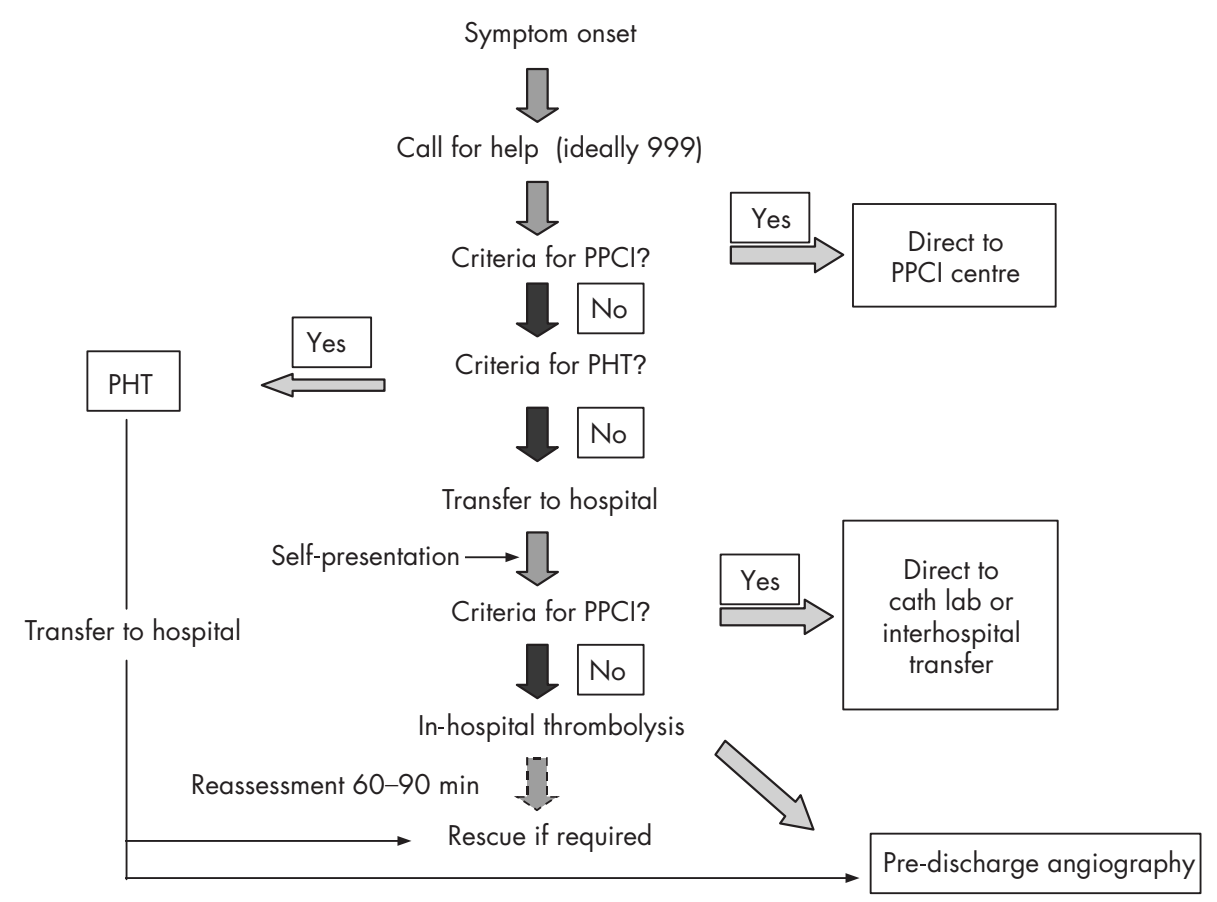

Figure 4 Stage 4 in the evolution of an emergency ST elevation myocardial infarction protocol. The optimum reperfusion pathway. Robust prehospital thrombolysis (PHT), primary percutaneous coronary intervention (PPCI), rescue percutaneous coronary intervention (PCI). 
PPCI is assumed and the only factors influencing which option is most appropriate relate to that particular patient at that particular time.

Although it would not be considered an "emergency" intervention, pre-discharge angiography should ideally, as discussed earlier, be performed in all patients who have had pharmacological reperfusion as their preferred initial strategy, irrespective of the apparent success of thrombolysis-this is included in fig 4 as part of the optimum reperfusion pathway.

\section{CONCLUSION}

Although proponents for interventional and pharmacological approaches for STEMI have sought to show that one is clearly better than the other, the current evidence base would suggest that no single approach would be beneficial for all patients in all situations. PHT, in-hospital thrombolysis and PPCI currently all have their place in the emergency treatment of patients with STEMI. Developing an infrastructure to enable robust access to PPCI $24 \mathrm{~h}$ a day is an appropriate ideal, but this is not the same as having no alternative for selected patients for whom the current evidence base would suggest that immediate pharmacological reperfusion would be more beneficial. The ideal scenario for any individual patient would be to have robust access to each therapeutic option, so that the clinician (whether a paramedic, emergency physician or cardiologist) could decide which would be most beneficial for their patient at that time. Such a nirvana of care is unlikely to arise in the near future and, until it does, we should continue to develop emergency care protocols that permit clinicians to tailor their treatments to their patients' clinical circumstances, the available resources and according to the latest evidence base.

This decision framework is presented as a tool that is evidence based, it combines pharmacological and interventional approaches, and considers both resource and clinical criteria in the development of reperfusion strategies for patients with STEMI.

Funding: This research was sponsored by an unrestricted educational grant from Boehringer Ingelheim.

Competing interests: None declared.

This paper represents the position of the Faculty of Pre-Hospital Care of the Royal College of Surgeons of Edinburgh, Edinburgh, UK.

\section{REFERENCES}

1 Thrombolysis Update. The ASA/JRCALC Clinical Effectiveness Programme 2006

2 MINAP Steering Group. How the NHS manages heart attacks. Fifth Public Report 2006.
3 Keeley EC, Boura JA, Grines CL. Primary angioplasty versus intravenous thrombolytic therapy for acute myocardial infarction: a quantitive review of 23 randomised trails. Lancet 2003;361:13-20.

4 Sutton A, Campbell PG, Graham R, et al. A randomized trial of rescue angioplasty versus a conservative approach for failed fibrinolysis in ST-segment elevation myocardial infarction: the Middlesbrough Early Revascularization to Limit INfarction (MERLIN) trial. J Am Coll Cardiol 2004;44:287-96.

5 Gershlick A, Stephens-Lloyd A, Hughes S, et al. Rescue angioplasty after failed thrombolytic therapy for acute myocardial infarction. N Engl J Med 2005;353:2758-68.

6 Bonnefoy E, Lapostolle F, Leizorovicz A, et al. Primary angioplasty versus prehospital thrombolysis in acute myocardial infarction: a randomized study. Lancet 2002;360:825-9.

7 Steg PG, Bonnefoy E, Chabaud S, et al. Impact of time to treatment on mortality after prehospital fibrinolysis or primary angioplasty. Data from the CAPTIM randomised clinical trial. Circulation 2003;108:2851-6.

8 Nallamothu BK, Bates ER. Percutaneous coronary intervention versus fibrinolytic therapy in acute myocardial infarction: is timing (almost) everything? Am J Cardiol 2003;92:824-6.

9 Magid D, Wang Y, Herrin J, et al. Relationship between time of day, day of week, timeliness of reperfusion, and in-hospital mortality for patients with acute STsegment elevation myocardial infarction. JAMA 2005;294:803-12.

10 Magid D, Calonge B, Rumsfeld J, et al. Relation between hospital primary angioplasty volume and mortality for patients with acute $\mathrm{Ml}$ treated with primary angioplasty vs thrombolytic therapy. JAMA 2000;284:3131-8.

11 The Task Force for Percutaneous Coronary Interventions of the European Society of Cardiology. Guidelines for percutaneous coronary interventions. Eur Heart J 2005;26:804-47.

12 The Task Force on the Management of Acute Myocardial Infarction of the European Society of Cardiology. Management of acute myocardial infarction in patients presenting with ST-segment elevation. Eur Heart J 2003;24:28-66.

13 Smith SC Jr, Dove JT, Jacobs AK, et al. ACC/AHA guidelines for percutaneous coronary intervention (revision of the 1993 PTCA guidelines). A report of the American College of Cardiology/American heart Association Task Force on Practice Guidelines. J Am Coll Cardiol 2001;37:2215-38.

14 American College of Cardiology/American Heart Association Task Force on Practice Guidelines. ACC/AHA Guidelines for the management of patients with ST-elevation myocardial infarction. Circulation 2004;110:588-636.

15 Hochman JS, Sleeper LA, Webb JG, et al. Early revascularization in acute myocardial infarction complicated by cardiogenic shock. N Engl J Med 1999;341:625-34.

16 Morrison L, Verbeek PR, McDonald AC, et al. Mortality and pre-hospital thrombolysis for acute myocardial infarction: a meta-analysis. JAMA 2000;283:2686-92.

17 Todd I. JRCALC clinical practice guidelines. Coventry: University of Warwick, 2004.

18 Fernandez-Avilés $F$, Alonso J Castro-Beiras A, et al. Routine invasive strategy within 24 hours of thrombolysis versus ischaemia-guided conservative approach for acute myocardial infarction with ST-segment elevation (GRACIA-1): a randomised controlled trial. Lancet 2004;364:1045-53.

19 Le May MR, Labinaz M, Turek M, et al. Combined Angioplasty and Pharmacological Intervention Versus Thrombolysis Alone in Acute Myocardia Infarction (CAPITAL-AMI Study). J Am Coll Cardiol 2005;46:417-24.

20 Verheugt FW. Lyse now, stent later: the grace of GRACIA. Lancet 2004;364:1014-15.

21 Keeley E, Boura J, Grines C. Comparison of primary and facilitated percutaneous interventions in ST-segment elevation myocardial infarction: a quantitative review of randomised trials. Lancet 2006;367:579-88.

22 National Institute for Clinical Excellence. Final appraisal determination: drugs for early thrombolysis in the treatment of acute myocardial infarction. London, UK: National Institute for Clinical Excellence, 2002

23 Department of Health. Review of early thrombolysis, Faster and better treatment for heart attack patient. London, UK: Department of Health, 2003. 